Rodríguez-García, A. \& Arias-Gago, A.R. (2021). Uso metodológico docente y rendimiento lector del alumnado: análisis fundamentado en PISA lectura 2018. Revista Electrónica Interuniversitaria de Formación del Profesorado, 24(3), 149-165.

DOI: https://doi.org/10.6018/reifop.469921

\title{
Uso metodológico docente y rendimiento lector del alumnado: análisis fundamentado en PISA lectura 2018
}

\author{
Alejandro Rodríguez-García, Ana Rosa Arias-Gago \\ Universidad de León. León, España.
}

\section{Resumen}

Las investigaciones en las que se vincula el rendimiento académico en lectura del alumnado y la utilización de modelos didácticos por parte del profesorado se caracterizan por el carácter exiguo y dispar de los resultados. Esta investigación analizó la influencia y relación entre la utilización metodológica que realizan 13119 docentes de España y el rendimiento académico del alumnado español en PISA lectura 2018. Para este propósito, se implementó un diseño de investigación descriptivo-correlacional en el que se relacionaron los resultados del alumnado español obtenido en la prueba de PISA lectura 2018 con los resultados extraídos tras la administración del cuestionario UMEPE a los docentes de esos alumnos. Los resultados arrojaron relaciones significativas y una influencia positiva y lineal entre el uso de un modelo activo y el rendimiento del alumnado en lectura. Por el contrario, cuando se implementa un modelo tradicional no se produce influencia ni relación entre las variables. La aplicación ecléctica de ambos modelos puede generar un mayor o menor rendimiento del alumnado en lectura en función del contexto. Estos hallazgos se anteponen a la mayoría de la literatura vinculada y se posicionan junto a los estudios que asocian métodos y estrategias activas con un mayor rendimiento.

\section{Palabras clave}

Método de enseñanza; Rendimiento lector; PISA lectura 2018; España.

\section{Contacto:}

Alejandro Rodríguez-García, arodrg01@estudiantes.unileon.es, Facultad de Educación, despacho 148, Universidad de León, Campus Vegazana, León, 24701 (España) 


\title{
Teaching methodological usage and students' reading achievement: analysis based on PISA reading 2018
}

\begin{abstract}
The studies that relate the use of teaching models and student's achievement in reading are characterized by their recent and disparate nature. This research analyzed both the influence and relationship between methodological usage made by 13119 Spanish teachers and the achievement of Spanish students in PISA reading 2018. To this end, a descriptivecorrelational research design was implemented in which the results of Spanish students obtained in PISA reading test are related to the results obtained after UMEPE questionnaire administration to the teachers of those students. The results showed significant relationships and a positive and linear influence between the use of active models by teachers and students' achievement in reading. On the contrary, when a traditional model is implemented, there is no influence or relationship between the variables. The eclectic application of both models can generate more or less students' achievement in reading depending on the context. These findings taker precedence over most of the connected literature and rank alongside studies that associate active methods and strategies with higher achievement.
\end{abstract}

\section{Key words}

Teaching method; Reading achievement; PISA reading 2018; Spain.

\section{Introducción}

La relevancia de los docentes en el rendimiento académico del alumnado ha sido constatada y aceptada por el grueso de la comunidad educativa (Gil, Cordero \& Cristóbal, 2018; Gil-Flores \& García-Gómez, 2017). Esta relevancia no implica que exista claridad a la hora de establecer qué actuaciones deben de implementar los docentes para mejorar los procesos de enseñanza y aprendizaje del alumnado y, por ende, su rendimiento académico (Sälzer \& Roczen, 2018).

Son numerosos los trabajos que señalan al constructo de la metodología docente como un elemento esencial tanto del aprendizaje como del rendimiento del alumnado (FernándezMartín, 2020; Gamazo, Martínez-Abad, Olmos-Miguelañez \& Rodríguez-Conde, 2018; Rodríguez-García \& Arias-Gago, 2020; Sáiz-Manzanares \& Valdivieso-León, 2020). En la literatura especializada, aparecen 2 modelos diferenciados asociados a este constructo (Jiménez-Hernández, González-Ortiz \& Tornel-Abellán, 2020; Murphy, Eduljee, Croteau \& Parkman, 2020): el tradicional, en el que el docente es la figura principal y el alumnado aprende de una forma receptiva; y el activo, en el que el profesorado actúa como guía y el alumnado es el protagonista de sus procesos de aprendizaje.

Los modelos citados disponen de un carácter transversal que permiten su aplicación en diferentes ámbitos y disciplinas, por lo que la lectura no es una excepción. La Organización para la Cooperación y el Desarrollo Económico (OCDE, 2019a) define a la competencia lectora, como: "la capacidad de los estudiantes de comprender, emplear, valorar, reflexionar e interesarse por los textos escritos, para alcanzar unos objetivos, desarrollar un conocimiento potencial propio y participar en la sociedad" (p.14). 
Esta competencia es fundamental para el aprendizaje del alumnado a lo largo de la vida y, a nivel educativo, es considerada un elemento transversal que debe de trabajarse desde todas las materias (Brevik, 2019; Rogiers, Van Keer \& Merchie, 2020; Sánchez-Fuentes, Martín-Almaraz, Moreno-Medina \& Espada-Chavarría, 2018; Wigfield, Gladstone \& Turci, 2016). También es un factor asociado al rendimiento académico del alumnado de ahí a que, una de las pruebas del Programa de evaluación Internacional de los Estudiantes (PISA) (OCDE, 2019a, 2020), se vincule de forma específica a su valoración. El marco conceptual de las pruebas de evaluación lectora de PISA, se dirige hacia que el alumnado clasifique diferentes tipologías textuales, evalúe diferentes procesos lectores y aplique estrategias lectoras en diferentes tareas.

Actualmente, en la literatura vinculada al aprendizaje y desarrollo de la competencia lectora, existen diversas prácticas de renovación pedagógica en las que se aplican modelos de carácter activo (Amiama-Espaillat \& Mayor-Ruiz, 2017; Schaffner, Philipp \& Schiefele, 2016; Zasacka \& Bulkowski, 2017). En muchas casuísticas, el profesorado implementa estos modelos activos y sus estrategias afines, sin tener evidencia empírica de que su utilización genera mejoras en el rendimiento académico de los discentes (Pérez-Pueyo \& Hortigüela, 2020; Ruiz-Marín, 2020).

Tanto a nivel nacional como internacional, la evidencia de la efectividad de este tipo de prácticas -enmarcadas en un modelo didáctico activo- en el rendimiento académico del alumnado en la competencia lectora, es bastante limitada como consecuencia de un reducido número de fuentes centradas en abordar la temática (Álvarez-Morán, Carleos, Corral \& Prieto, 2018; Gamazo et al., 2018; Gil et al., 2018). A la problemática mencionada, se añade que las evaluaciones estandarizadas -nacionales e internacionales- centradas en evaluar y vincular el rendimiento académico del alumnado en la competencia lectora son relativamente recientes (primera medición de PISA lectura en el año 2002) (Álvarez-Morán et al., 2018; Lorenzo, 2016; Van Hek, Kraaykamp \& Pelzer, 2018).

A las problemáticas del carácter reciente y novedoso de la evaluación del rendimiento académico del alumnado en la competencia lectora y de la escasez de evidencias empíricas en la literatura se añade, según Álvarez-Morán et al. (2018) y O’Connell (2019), la disparidad existente entre los resultados de las investigaciones, en las que en unos casos el mayor uso del modelo activo (Comi, Argentin, Gui, Origo \& Pagani, 2017; Huang, Tang, He \& Li, 2019; Petko, Cantieni \& Prasse, 2017, Rogiers et al., 2020; Tavsancil, Yildirim \& Bilican, 2019) y en otros el mayor uso del tradicional (Brevik \& Hellekjær, 2018; Fernández-Gutiérrez, Gimenez \& Calero, 2020; Gubbels, Swart \& Groen, 2020; Lau \& Ho, 2016; Perera \& Asadullah, 2019; Vázquez-Cano, Gómez-Galán, Infante-Moro \& López-Meneses, 2020), se asocia a un mayor rendimiento lector del alumnado.

Las investigaciones citadas tienen como aspecto común que el rendimiento es cuantificado a través del programa de Evaluación Internacional de Estudiantes (PISA). En la presente investigación, con la finalidad de usar un marco de medición común con la literatura, se usaron los resultados que aparecen en el informe español PISA 2018 (OCDE, 2019a) para la competencia lectora (OCDE, 2020).

A pesar de este aspecto común, las problemáticas enunciadas -disparidad en los resultados de las investigaciones, aplicación reciente de pruebas internacionales de evaluación de la competencia lectora, y escasez de estudios que relacionen el rendimiento académico en la competencia lectora y la utilización de modelos didácticos- subrayan la necesidad de implementar nuevas investigaciones con los datos del reciente informe PISA 2018 (OCDE, 2019a, 2020), para cuantificar en el contexto español la utilización de modelos tradicionales 
y activos por parte del profesorado y, de esta forma, establecer qué modelo influye más en la competencia lectora del alumnado.

Siguiendo este propósito, la investigación presentó 2 objetivos diferenciados: (a) describir el uso que los docentes de España realizan de los principales modelos de enseñanza -activo y tradicional- asociados al desarrollo de la competencia lectora y (b) establecer cómo el uso de uno u otro influye en el desempeño académico del alumnado en la competencia lectora.

\section{Metodología}

\section{Participantes}

Se utilizó un muestreo aleatorio por conglomerados bietápico para la selección de docentes. En la primera etapa, se dividió la población objeto de estudio en 17 estratos, los cuales se corresponden con las 17 comunidades autónomas del estado español y en cada estrato, se consideraron como conglomerados los centros participantes en PISA 2018. En la segunda etapa, se administró el cuestionario UMEPE (Uso de métodos de enseñanza en el profesorado español) a los docentes que han impartido docencia a grupos de alumnado participante en PISA 2018.

La muestra, representativa de la población $(n=257659)(M E F P, 2020 a)$ con un margen de error del $2 \%$ y un nivel de confianza del $99 \%$, se compuso por un total de $13119(n=13119)$ docentes de la etapa de Educación Secundaria Obligatoria (ESO) (tabla 1), con el requisito prescriptivo de que hayan impartido docencia a grupos de alumnado participante en PISA 2018. La inclusión de docentes de todas las asignaturas de ESO está motivada por la transversalidad de la lectura, que hace que a nivel curricular deba de ser tratada desde todas las materias.

Tabla 1.

Población y muestra de docentes por comunidad autónoma

\begin{tabular}{lll}
\hline Comunidad autónoma & Población & $\mathbf{n}$ \\
\hline Castilla y León & 13244 & 690 \\
\hline Asturias & 5353 & 275 \\
\hline Galicia & 14811 & 742 \\
\hline Aragón & 7888 & 398 \\
\hline Cataluña & 38032 & 1934 \\
\hline Cantabria & 4213 & 215 \\
\hline Murcia & 8706 & 443 \\
\hline Islas Baleares & 6314 & 321 \\
\hline Castilla La Mancha & 13399 & 676 \\
\hline País Vasco & 12582 & 630 \\
\hline
\end{tabular}




\begin{tabular}{lll}
\hline Comunidad autónoma & Población & $\mathbf{n}$ \\
\hline Madrid & 24148 & 1234 \\
\hline Comunidad Valenciana & 29739 & 1493 \\
\hline Navarra & 4339 & 220 \\
\hline Canarias & 12641 & 652 \\
\hline La Rioja & 1693 & 110 \\
\hline Andalucía & 52453 & 2675 \\
\hline Extremadura & 8104 & 411 \\
\hline Total & 257659 & 13119 \\
\hline
\end{tabular}

Nota. Fuente: elaboración propia a partir del MEFP (2020a)

Por otra parte, se consultaron fuentes de información vinculadas a la base de datos PISA 2018, en la que la muestra total para el alumnado de 15 y 16 años de España ascendió a 35943 ( $n=35943$ ) (OCDE, 2019a). Las características del tipo de muestreo utilizado se encuentra desarrollado en el documento denominado PISA 2018 Technical Report (OCDE, 2019b).

\section{Instrumento}

Para la construcción del cuestionario se partió de un instrumento previo denominado OPPUMAEOL (Rodríguez-García \& Arias-Gago, 2019), adaptándose los diferentes ítems al contexto nacional de la investigación. También se consultaron otros instrumentos vinculados de la literatura asociada como el inventario ATI de enfoques de enseñanza (Trigwell \& Prosser, 2004) y el cuestionario para la medición de métodos activos en el ámbito universitario (Jiménez-Hernández et al., 2020).

Este proceso de revisión y modificación posibilitó la génesis del cuestionario UMEPE, compuesto por 3 partes diferenciadas: 1) centrada en el abordaje de aspectos sociodemográficos 2) compuesta por una escala en la que los docentes valoraron de 0 a 3 puntos (o-nada, 1-poco, 2-bastante, 3-mucho) la utilización y conocimiento de los modelos tradicionales y activos 3) compuesta por otra escala centrada en valorar las percepciones y opiniones de estos docentes hacia el uso de modelos activos.

El cuestionario fue validado a través del procedimiento denominado método Delphi, en el que 9 expertos -profesores-doctores en Ciencias de la Educación con al menos 5 años de experiencia- valoraron la relevancia, pertinencia y univocidad de cada ítem, proponiendo modificaciones cuando los ítems no se ajustaban a los criterios citados. Para finalizar la validación, el cuestionario se administró en la muestra de la investigación. Tras la recogida de datos, se llevó a cabo un análisis factorial confirmatorio [CFI=.921 ] y un análisis factorial exploratorio $[\mathrm{KMO}=.827)]$, cuyos resultados, una vez suprimidos 5 ítems, arrojaron unas propiedades psicométricas óptimas para los 92 ítems del cuestionario, de los cuales solamente se utilizaron los vinculados con la investigación. 
La fiabilidad del cuestionario en su totalidad fue hallada por medio del coeficiente Alfa de Cronbach [ $\mathrm{R}=.822$ ] y la varianza promedio extraída [AVE=.593], en las que los valores obtenidos fueron óptimos (Gaitán \& Piñuel, 1997).

\section{Procedimiento}

En primer lugar, se agrupó a la población de docentes de ESO en 17 estratos, que se corresponde con las 17 comunidades autónomas del territorio español. Posteriormente, en cada estrato, se establecieron conglomerados que se corresponden con los Institutos de Enseñanza Secundaria (IES) participantes en PISA 2018. Para conocer los IES participantes, se consultó el PISA 2018 technical report (OCDE, 2019b) y posteriormente, por medio del registro español de centros docentes no universitarios (MEFP, 2020b), se recopilaron los correos electrónicos de esos IES.

Tras la recopilación, el cuestionario fue digitalizado utilizando el programa Microsoft Forms y enviado a los directores de los IES participantes en PISA 2018 con una carta en la que se explicaba el propósito de la investigación y el proceso a implementar para la administración del mismo a los profesores de cada IES. En la citada carta, se hizo hincapié en que el equipo directivo solo administrase el cuestionario a los profesores de grupos de alumnos que hayan participado en PISA 2018, de esta forma y con un ítem específico del cuestionario en el que se pregunta a los docentes, se aseguró que la muestra ha impartido docencia a alumnado participante en PISA lectura 2018. De esta manera, los equipos directivos de los centros educativos que decidieron participar, reenviaron el cuestionario a los profesores, quienes respondieron al mismo de forma on-line, anónima y voluntaria en el periodo comprendido entre noviembre de 2019 y marzo de 2020.

\section{Diseño de investigación y análisis de datos}

Se siguió un diseño de investigación cuantitativo no experimental de tipo descriptivo correlacional y transversal (Colás, Buendía \& Hernández, 2009). El análisis de datos se llevó a cabo utilizando la versión 26 del software SPSS, implementándose los siguientes análisis: en la parte descriptiva, se aplicó un análisis de las puntuaciones promedio obtenidas por docentes en el cuestionario UMEPE y por el alumnado en PISA lectura 2018; y en la parte regresiva y correlacional, se implementó un análisis de regresión lineal y un análisis de correlación bivariado con los que observar por un lado, cómo una variable influye sobre otra y, por otro, cómo se relacionan.

\section{Resultados}

Los resultados han sido extraídos tanto por medio de un análisis descriptivo como por medio de un análisis regresivo y correlacional. Es necesario puntualizar que, aunque se hable de utilización metodológica a lo largo del trabajo por parte de los docentes, lo que se midió es la auto-percepción docente hacia la utilización de modelos didácticos.

\section{Análisis descriptivo}

Estos análisis se han dirigido a desarrollar el objetivo vinculado a describir cómo es la utilización que los docentes de las diferentes comunidades autónomas efectúan tanto del modelo tradicional como del activo, así como a describir el rendimiento académico del alumnado de esas comunidades autónomas en PISA lectura 2018. En este análisis, de tipología descriptiva, se utilizaron puntuaciones promedio como principal índice de medición. 
Para la delimitación cualitativa del rendimiento académico del alumnado en PISA lectura 2018, la OCDE (2019a) estableció los siguientes niveles de rendimiento promedio: nivel 1, para alumnado con puntuaciones entre 0 y 335; nivel 2, con puntuaciones comprendidas entre 336 y 406; nivel 3, puntuaciones entre 407 y 479; nivel 4, puntuaciones entre 480 y 552; y niveles 5 y 6 , para puntuaciones superiores, no detalladas al no ser alcanzadas por el alumnado. De forma similar, para la delimitación cualitativa de las puntuaciones obtenidas por el profesorado en la utilización de los modelos didácticos, se diseñó un sistema de categorías exhaustivo y mutuamente excluyente, en el que se integraron los siguientes niveles de puntuaciones promedio: nivel alto, para puntuaciones entre 2.01 y 3 ; nivel medio, para puntuaciones entre 1.01 y 2; y nivel bajo, para puntuaciones ubicadas entre 0 y 1.

Tal y como aparece reflejado en la tabla 2, el alumnado de las comunidades autónomas de Castilla y León [ $\bar{x}=497$ ], Asturias [ $\bar{x}=495]$, Galicia [ $\bar{x}=494$ ], Aragón [ $\bar{x}=490]$, Cataluña [ $\bar{x}=483$ ], Cantabria $[\bar{x}=483]$ y Murcia $[\bar{x}=481]$ se encuentran en el nivel 4 de PISA lectura, aspecto que implica que son capaces de comprender y razonar de forma causal sobre diversos textos extensos, inferir la información relevante de los mismos, distinguir entre hechos y opiniones, y extraer conclusiones de enunciados complejos (OCDE, 2019a). En el nivel 3, se sitúa el alumnado de las comunidades autónomas de Islas Baleares [ $\bar{x}=479]$, Castilla La Mancha [ $\bar{x}=478$ ], País Vasco [ $\bar{x}=475]$, Madrid [ $\bar{x}=474]$, Comunidad Valenciana [ $\bar{x}=473]$, Navarra [ $\bar{x}=472$ ], Islas Canarias [ $\bar{x}=472]$, La Rioja [ $\bar{x}=467]$, Andalucía [ $\bar{x}=466$ ] y Extremadura $[\bar{x}=464]$, lo que indica que pueden representar el sentido literal de varios textos, generar inferencias básicas, detectar ideas principales y secundarias, entender relaciones y comparar y contrastar información explícita de varios textos (OCDE, 2019a).

En las medidas de utilización de modelos didácticos (tabla 2), los docentes de las comunidades autónomas obtienen valores promedio de utilización del modelo tradicional ubicadas en un nivel alto de utilización. En este sentido, todas las comunidades disponen de puntuaciones por encima de $\bar{x}=2.3$, salvo Castilla y León [ $\bar{x}=2.2]$, La Rioja [ $\bar{x}=2.11$ ], Castilla La Mancha $[\bar{x}=2.09]$ y Extremadura $[\bar{x}=2.08]$, que disponen de puntuaciones inferiores.

\section{Tabla 2.}

Promedio de puntuaciones del alumnado en PISA lectura y del profesorado en la utilización de modelos didácticos

\begin{tabular}{llll}
\hline Comunidad autónoma & $\overline{\mathbf{x}}$ PISA lectura & $\overline{\mathbf{x}}$ modelo tradicional & $\overline{\mathbf{x}}$ modelo activo \\
\hline Castilla y León & 497 & 2.2 & 2.31 \\
\hline Asturias & 495 & 2.36 & 1.95 \\
\hline Galicia & 494 & 2.31 & 2.58 \\
\hline Aragón & 490 & 2.33 & 2.14 \\
\hline Cataluña & 484 & 2.41 & 1.71 \\
\hline Cantabria & 483 & 2.31 & 2.09 \\
\hline Murcia & 481 & 2.44 & 1.33 \\
\hline Islas Baleares & 479 & 2.48 & 1.73 \\
\hline
\end{tabular}




\begin{tabular}{llll}
\hline Comunidad autónoma & $\overline{\mathbf{X}}$ PISA lectura & $\overline{\mathbf{x}}$ modelo tradicional & $\overline{\mathbf{x}}$ modelo activo \\
\hline Castilla La Mancha & 478 & 2.09 & 1.52 \\
\hline País Vasco & 475 & 2.3 & 2 \\
\hline Madrid & 474 & 2.43 & 1.5 \\
\hline Comunidad Valenciana & 473 & 2.33 & 1 \\
\hline Navarra & 472 & 2.36 & 2.22 \\
\hline Canarias & 472 & 2.39 & 0.9 \\
\hline La Rioja & 467 & 2.11 & 2.06 \\
\hline Andalucía & 466 & 2.37 & 1.06 \\
\hline Extremadura & 464 & 2.08 & 1.21 \\
\hline Promedio España & 479.06 & 2.31 & 1.72 \\
\hline
\end{tabular}

En el modelo activo, tal y como puede observarse en la tabla 2, los docentes de las comunidades autónomas de Galicia [ $\bar{x}=2.58]$, Castilla y León $[\bar{x}=2.31]$, Navarra $[\bar{x}=2.22]$, Aragón [ $\bar{x}=2.14]$, Cantabria $[\bar{x}=2.09]$ y La Rioja $[\bar{x}=2.06]$ se categorizan en un nivel alto de utilización. Por su parte, docentes de comunidades autónomas como País Vasco [ $\bar{x}=2]$, Asturias [ $\bar{x}=1.95$ ], Islas Baleares [ $\bar{x}=1.73]$, Cataluña [ $\bar{x}=1.71$ ], Castilla La mancha $[\bar{x}=1.52]$, Madrid [ $\bar{x}=1.5]$, Murcia [ $\bar{x}=1.33]$, Extremadura [ $\bar{x}=1.21]$ y Andalucía [ $\bar{x}=1.06]$, se sitúan en un nivel medio. De forma opuesta, docentes de la Comunidad Valenciana $[\bar{x}=1]$ e Islas Canarias $[\bar{x}=0.9]$ se ubican en un nivel de utilización bajo.

\section{Análisis regresivo y correlacional}

Los presentes análisis se han enfocado en el desarrollo del objetivo vinculado a determinar la relación e influencia entre el uso de modelos didácticos -activo y tradicional- que efectúa el profesorado de ESO de las diferentes comunidades autónomas y el rendimiento académico del alumnado de esas comunidades en PISA lectura.

Para establecer relaciones entre la variable utilización de un modelo activo por el profesorado y el rendimiento académico del alumnado en lectura, se aplicó un modelo regresivo lineal, en el que se partió de una hipótesis nula de independencia lineal entre las variables. En la tabla 3, la dependencia lineal entre las variables fue cotejada por medio del coeficiente de determinación $\mathrm{R}^{2}=.389$, el cual indica que el uso de un modelo activo por parte de los docentes explica el rendimiento académico del alumnado en el 38.9\% de los casos. Por su parte, el coeficiente $\mathrm{R}^{2}$ ajustado dispuso de un valor de $34.8 \%$ aspecto que, considerando a Calderón, Arias-Estero, Meroño y Méndez-Giménez (2018), implica una bondad de ajuste buena para el campo de las Ciencias Sociales, ya que los valores son >.3 . 


\section{Tabla 3.}

Regresión lineal y ANOVA del modelo para la variable rendimiento académico en PISA lectura 2018 con la utilización de un modelo activo

\begin{tabular}{|c|c|c|c|c|c|c|c|c|}
\hline \multirow{2}{*}{ Modelo } & \multirow{2}{*}{$\mathbf{R}$} & \multirow{2}{*}{$\mathbf{R}^{2}$} & \multirow{2}{*}{$\begin{array}{l}\mathbf{R}^{2} \\
\text { ajustado }\end{array}$} & \multirow{2}{*}{ Error } & \multicolumn{4}{|c|}{ ANOVA } \\
\hline & & & & & F & Gl1 & Gl2 & Sig. \\
\hline $\begin{array}{l}\text { Uso } \\
\text { modelo } \\
\text { activo }\end{array}$ & $.624^{a}$ & .389 & .348 & 8.285 & 9.546 & 1 & 15 & $.007^{* *}$ \\
\hline
\end{tabular}

La hipótesis nula se contrastó por medio de un análisis ANOVA con el estadístico de contraste $\mathrm{F}=9.546$, cuyos valores arrojaron una significación <.01 (sig.=.007). Este análisis ha permitido rechazar la hipótesis nula de independencia lineal, debido a que las variables objeto de análisis están influenciadas de forma significativa, aspecto que implica un ajuste del modelo bueno con valores inferiores a .05 .

Los coeficientes regresivos aparecen en la tabla 4 y muestran el cambio medio que le supone a la variable rendimiento académico en PISA lectura 2018 por cada unidad de cambio en la variable utilización de un modelo activo por parte del profesorado. Estos coeficientes no estandarizados aparecen en la fórmula regresiva de la figura 1, en la que se integra el promedio de las puntuaciones de las diferentes comunidades autónomas tanto en la utilización del modelo activo como en el rendimiento académico del alumnado. Por su parte, el estadístico $t$, establece una relación directamente proporcional y significativa tanto para la variable utilización de un modelo activo [t=3.090-Sig.=.007] como para la variable rendimiento académico del alumnado en PISA lectura 2018 [ $\mathrm{t}=61.779$-Sig.=.000].

\section{Tabla 4.}

Coeficientes de regresión y correlaciones del modelo activo para la variable rendimiento académico del alumnado en PISA lectura

\begin{tabular}{|c|c|c|c|c|c|c|}
\hline \multirow{2}{*}{ Modelo } & \multicolumn{2}{|c|}{$\begin{array}{l}\text { Coeficientes no } \\
\text { estandarizados }\end{array}$} & \multirow{2}{*}{$\begin{array}{c}\begin{array}{c}\text { Coeficientes } \\
\text { estandarizados }\end{array} \\
\text { Beta }\end{array}$} & \multirow{2}{*}{$\mathbf{t}$} & \multirow{2}{*}{ Sig. } & \multirow{2}{*}{ Correlaciones } \\
\hline & B & $\begin{array}{l}\text { Error } \\
\text { estándar }\end{array}$ & & & & \\
\hline \multirow{2}{*}{$\begin{array}{l}\text { Uso } \\
\text { modelo } \\
\text { activo }\end{array}$} & 457.06 & 7.398 & \multirow{2}{*}{.624} & 61.779 & .000 & \multirow{2}{*}{$.624^{* *}$} \\
\hline & 12.760 & 4.130 & & 3.090 & .007 & \\
\hline
\end{tabular}

El análisis de correlación corrobora la dependencia lineal entre las variables establecidas en el modelo regresivo e indica valores positivos y significativos a través del coeficiente $R$ de Pearson [R=624-sig.=.004], ya que el valor de $R$ es >.5 (Calderón et al., 2018). 
Figura 1.

Análisis de regresión por comunidades autónomas y variables analizadas

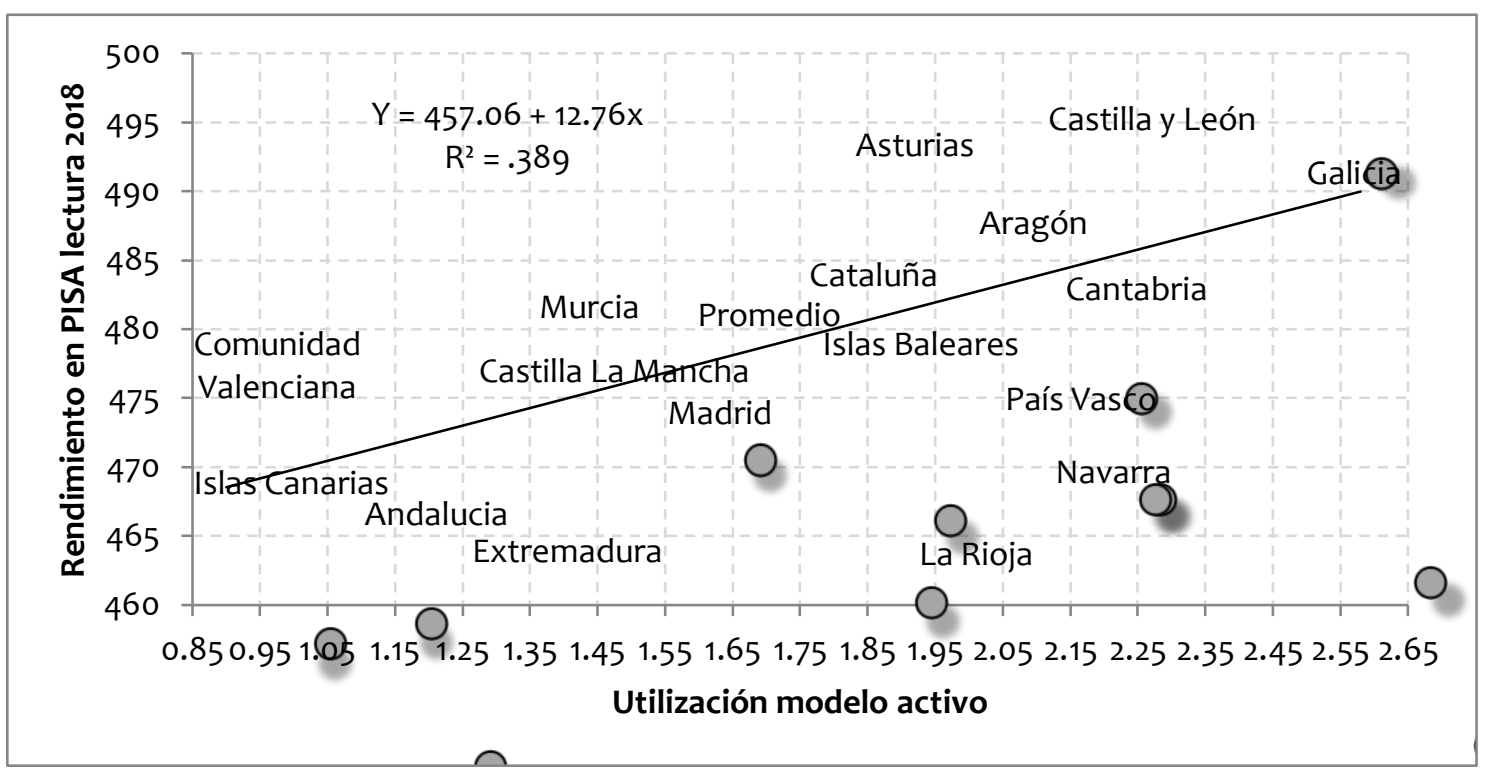

El modelo regresión implementado se muestra en la figura 1, en la que se observa la relación e influencia entre el rendimiento académico del alumnado en PISA lectura 2018 y la utilización de un modelo activo por parte de los docentes, utilizando como variable de agrupación a las diferentes comunidades autónomas.

Para determinar la relación entre la utilización de un modelo tradicional y el rendimiento académico del alumnado en PISA lectura 2018, se aplicó un nuevo análisis de regresión lineal. Como sucedió en el caso anterior, se partió de una hipótesis nula de independencia en la linealidad entre las variables citadas. El modelo aparece reflejado en la tabla 5 , donde el coeficiente de determinación de $\mathrm{R}^{2}=.030$ indica que la utilización de un modelo tradicional por parte de los docentes explica el rendimiento académico del alumnado en lectura en un $3 \%$ de las casuísticas. El $\mathrm{R}^{2}$ ajustado arrojó un valor de $-3.4 \%$, lo que indica, según Calderón et al. (2018), que las variables apenas se relacionan e influyen una sobre otra y, cuando lo hacen, es de forma negativa.

Tabla 5.

Regresión lineal y ANOVA del modelo para la variable rendimiento académico en PISA lectura 2018 con la utilización de un modelo tradicional

\begin{tabular}{|c|c|c|c|c|c|c|c|c|}
\hline \multirow{2}{*}{ Modelo } & \multirow{2}{*}{$\mathbf{R}$} & \multirow{2}{*}{$\mathbf{R}^{2}$} & \multirow{2}{*}{$R^{2}$ ajustado } & \multirow{2}{*}{ Error } & \multicolumn{4}{|c|}{ ANOVA } \\
\hline & & & & & $\mathbf{F}$ & Gl1 & $\mathrm{Gl} 2$ & Sig. \\
\hline $\begin{array}{l}\text { Uso modelo } \\
\text { tradicional }\end{array}$ & $.174^{a}$ & .030 & -.034 & 10.436 & .469 & 1 & 15 & .504 \\
\hline
\end{tabular}

Nota. a=predictores: (constante), uso modelo activo 
En este caso, se aceptó la hipótesis nula de independencia lineal entre las variables ya que, el análisis ANOVA implementado a través del estadístico $\mathrm{F}=.469$, arrojó valores no significativos (sig.=.504). En este sentido, una significación superior a .05 supone la aceptación de la hipótesis nula.

Los coeficientes de regresión no estandarizados de la recta aparecen reflejados en la tabla $6 \mathrm{y}$, en este caso, muestran una proporción significativa y directa para el rendimiento académico del alumnado en PISA lectura 2018 [ $\mathrm{t}=9.019$-sig.=.000], y positiva y no significativa para la utilización de un modelo tradicional por parte del profesorado [ $\mathrm{t}=.685$ sig.=.504]. Estos resultados indican una relación débil y no significativa entre la utilización de un modelo tradicional y el rendimiento académico del alumnado.

\section{Tabla 6.}

Coeficientes de regresión y correlaciones del modelo tradicional para la variable rendimiento académico del alumnado en PISA lectura

\begin{tabular}{|c|c|c|c|c|c|c|}
\hline \multirow{2}{*}{ Modelo } & \multicolumn{2}{|c|}{$\begin{array}{l}\text { Coeficientes no } \\
\text { estandarizados }\end{array}$} & \multirow{2}{*}{$\begin{array}{c}\begin{array}{c}\text { Coeficientes } \\
\text { estandarizados }\end{array} \\
\text { Beta }\end{array}$} & \multirow{2}{*}{$t$} & \multirow{2}{*}{ Sig. } & \multirow{2}{*}{ Correlaciones } \\
\hline & B & $\begin{array}{l}\text { Error } \\
\text { estándar }\end{array}$ & & & & \\
\hline \multirow{2}{*}{$\begin{array}{l}\text { Uso modelo } \\
\text { tradicional }\end{array}$} & 445.285 & $49 \cdot 371$ & \multirow{2}{*}{.174} & 9.019 & .000 & \multirow{2}{*}{.174} \\
\hline & 14.61 & 21.328 & & .685 & .504 & \\
\hline
\end{tabular}

\footnotetext{
Significación $\mathrm{p}<.01$
}

El análisis de correlación aplicado a través del coeficiente $R$ de Pearson=.174, indica una relación débil y no significativa (sig.=.252) entre las variables del modelo (Calderón et al., 2018), por lo que los datos no se ajustan de forma óptima al modelo regresivo utilizado.

Figura 2.

Análisis de regresión por comunidades autónomas y variables analizadas

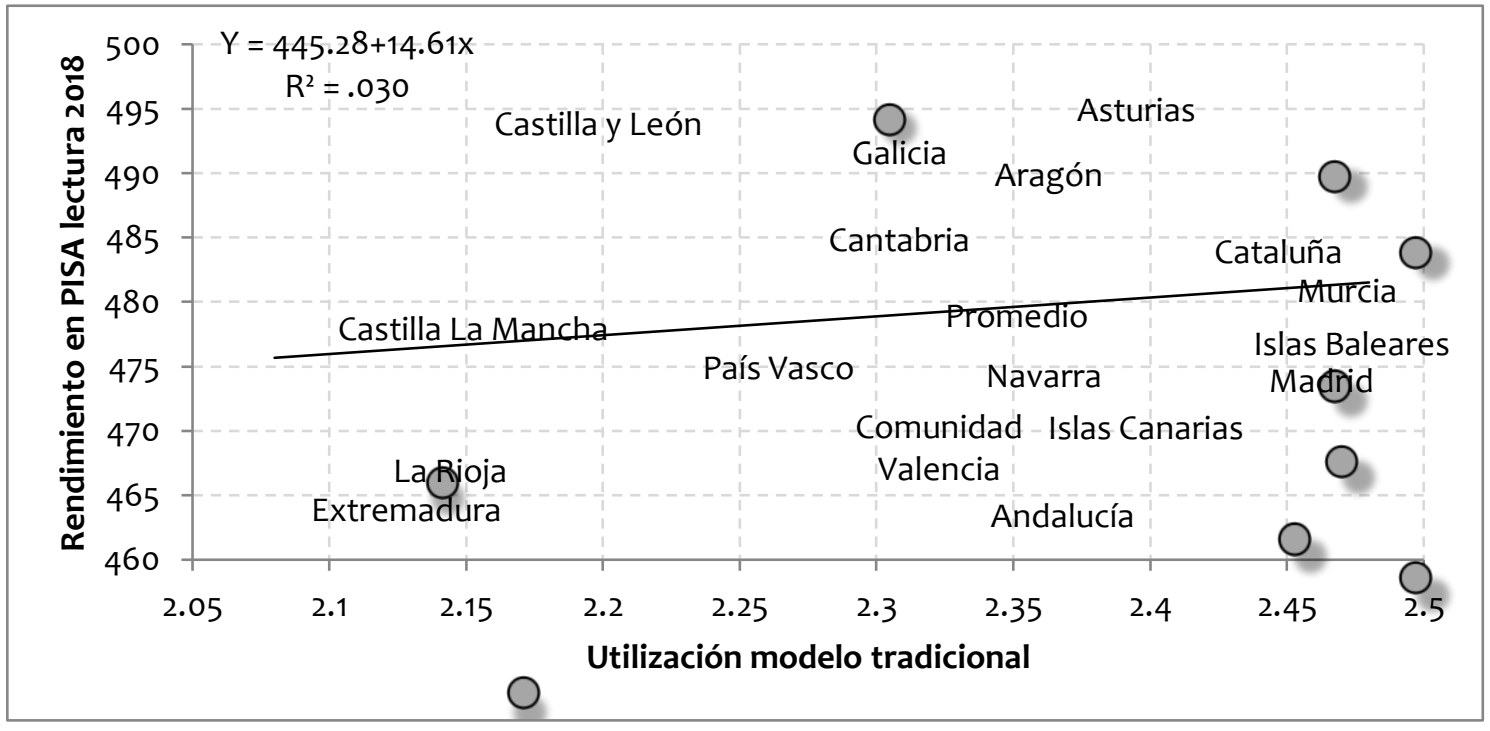


El modelo regresivo implementado aparece reflejado en la figura 2, en la que se observa una gran dispersión entre las puntuaciones promedio de las diferentes comunidades autónomas, así como una escasa relación e influencia entre el uso de un modelo tradicional por parte del profesorado y el rendimiento académico del alumnado en PISA lectura 2018.

\section{Discusión y conclusiones}

Los resultados de la presente investigación han permitido determinar la existencia de una influencia lineal y positiva entre el uso de un modelo activo por parte de los docentes y el rendimiento académico del alumnado de las diferentes comunidades autónomas en PISA lectura 2018. Por el contrario, cuando los docentes utilizan un modelo tradicional, la relación e influencia entre las variables es exigua y dispersa.

Esta diferenciación en la relación e influencia entre las variables también se traslada a la utilización promedio de los diferentes modelos didácticos por parte del profesorado de las diferentes comunidades autónomas. A este respecto, la utilización metodológica por parte de los docentes de las diferentes comunidades, sitúa al modelo activo en los 3 niveles de utilización establecidos (bajo, medio y alto) y al tradicional, en un nivel alto.

La variabilidad citada se traslada, también, a las puntuaciones promedio del desempeño académico del alumnado en PISA lectura 2018. Islas Baleares, Castilla La Mancha, País Vasco, Madrid, Comunidad Valenciana, Navarra, Islas Canarias, La Rioja, Andalucía y Extremadura se ubican en el nivel 3 (puntuaciones promedio entre 407 y 479) y las comunidades de Castilla y León, Asturias, Galicia, Aragón, Cataluña, Cantabria y Murcia en el 4 (puntuaciones promedio entre 480 y 552 ).

También es destacable que el alumnado de comunidades autónomas como Galicia, Aragón, Castilla y León y Cantabria obtiene las puntuaciones más altas, ubicadas en un nivel 4 en PISA lectura, y la utilización que efectúan los docentes se sitúa en un nivel alto tanto en el modelo tradicional como en el activo. Este hallazgo sucede, según Harks, Rakoczy, Hattie, Besser y Klieme (2014), porque la utilización ecléctica de modelos activos y tradicionales no impide el rendimiento académico del alumnado y, en función del contexto, en unos casos puede potenciarlo y en otros disminuirlo (Algan, Cahuc \& Shleifer, 2013).

Pese al eclecticismo de las comunidades mencionadas, la tendencia más común se vincula a que en el modelo tradicional, en el que el docente instruye y al alumnado adquiere los contenidos de forma pasiva, los resultados se caracterizaron por un lado, por la escasa influencia y relación entre las variables y, por otro, por una gran dispersión entre las mismas. Este hallazgo se contrapone a la mayoría de la literatura vinculada a la temática objeto de estudio en la que, en la mayoría de casuísticas, la utilización de modelos y métodos tradicionales se asocia a un mayor rendimiento académico del alumnado en PISA lectura (Dong \& Hu, 2019; Giménez, Barrado \& Arias, 2019; Gubbels et al., 2020; Perera \& Asadullah, 2019; Torrpa, Eklund, Sulkunen, Niemi \& Ahonen, 2017).

De forma opuesta, la investigación arroja que, el uso de un modelo activo por parte de los docentes, se relaciona significativamente e influye de forma lineal y directa en el rendimiento académico del alumnado en lectura. Esta circunstancia, de forma similar al caso anterior, aparece en algunas investigaciones de la literatura (Alderete, Di Meglio \& Formichella, 2017; Meroni, Vera-Toscano \& Costa, 2015; Rogiers et al., 2020; Tavsancil et al., 2019) si bien, el número de fuentes es menor que en el caso anterior. 
Los hallazgos obtenidos deben de ser interpretados con cierta cautela, debido a que no pueden ser valorados en términos de causalidad. El rendimiento académico del alumnado no está solamente influenciado por la metodología docente, sino que influyen otros factores -contexto socio-económico, políticas educativas, centro escolar, factores asociados al estudiante, factores familiares y ambientales, desempeño y desarrollo docente, factores emocionales, factores tecnológicos, etcétera- (Amiama-Espaillat \& Mayor-Ruiz, 2018; Gil et al., 2018; Gil-Flores \& García-Gómez, 2017; Martínez-Oviedo, ToledoToledo \& Martínez-Mendoza, 2021; Vázquez-Cano, De-la-Calle-Cabrera, Hervás-Gómez \& López-Meneses, 2020).

La principal conclusión que se extrae de esta investigación se asocia por una parte, a la existencia de una influencia lineal y positiva entre la utilización de un modelo activo por parte del profesorado y el rendimiento académico del alumnado en lectura y, por otra, a la relación positiva y significativa entre ambas variables. Esta circunstancia no se traslada a la utilización de un modelo tradicional ya que, en este caso, la relación e influencia entre las variables es escasa. No obstante, si este modelo tradicional se aplica de forma ecléctica con un modelo activo, puede generar mayor o menor rendimiento académico del alumnado en lectura en función del contexto de aplicación. Los hallazgos de este estudio amplían la escasa literatura existente (Álvarez-Morán et al., 2018; Gil et al., 2018) y se sitúan junto con las investigaciones que vinculan la utilización de un modelo activo de enseñanza con un rendimiento académico del alumnado mayor en la competencia lectora.

Las citadas implicaciones no impiden que la investigación se encuentre exenta de limitaciones. En este sentido, la primera es inherente al método utilizado ya que, los análisis regresivos, se utilizan para identificar la influencia entre las variables objeto de estudio pero no permiten establecer relaciones de causalidad que doten de una mayor confiabilidad a los resultados obtenidos. Esta limitación abre una futura línea de investigación en la que se implemente un diseño cuasi-experimental aplicando los citados modelos didácticos en diversos grupos para valorar de forma causal el efecto de los mismos sobre el rendimiento del alumnado en lectura. La siguiente versa sobre el hecho de que solo se ha valorado el efecto que el uso de un modelo tradicional o activo tiene en el rendimiento del alumnado en lectura. Futuras investigaciones podrían centrarse en determinar qué métodos y estrategias docentes incluidas en el seno de cada modelo (activo y tradicional), generan mayor o menor rendimiento académico del alumnado. Todo ello, con la finalidad de facilitar la toma de decisiones de las diferentes administraciones educativas a la hora planificar e introducir estos modelos en los futuros currículos educativos de la LOMLOE para mejorar el aprendizaje y desarrollo lector del alumnado desde una perspectiva basada en la evidencia.

\section{Referencias}

Alderete, M. V., Di Meglio, G., \& Formichella, M. M. (2017). Acceso a las TIC y rendimiento educativo: ¿una relación potenciada por su uso? Un análisis para España. Revista de Educación, 377, 54-81. https://doi.org/10.4438/1988-592X-RE-2017-377-353

Algan, Y., Cahuc, P., \& Shleifer, A. (2013). Teaching practices and social capital. American Economic Journal: Applied Economics, 5(3), 189-210. https://doi.org/10.1257/app.5.3.189

Álvarez-Morán, S., Carleos, C.E., Corral, N.O., \& Prieto, E. (2018). Metodología docente y rendimiento en PISA 2015: Análisis crítico. Revista de Educación, 379, 85-114. https://doi.org/10.4438/1988-592X-RE-2017-379-370 
Amiama-Espaillat, C., \& Mayor-Ruiz, C. (2017). Lectura digital en la competencia lectora: La influencia en la Generación Z de la República Dominicana. Comunicar, 52(25), 105-114. https://doi.org/10.3916/C52-2017-10

Amiama-Espaillat, C., \& Mayor-Ruiz, C. (2018). Explorando la relación entre fluidez lectora y competencia lectora en Educación Secundaria. Ocnos, 17(1), 21-31. http://dx.doi.org/10.18239/ocnos_2018.17.1.1278

Brevik, L. M. (2019). Explicit reading strategy instruction or daily use of strategies? Studying the teaching of reading comprehension through naturalistic classroom observation in English L2. Reading and Writing, 32, 2281-2310. https://doi.org/10.1007/s11145-01909951-W

Brevik, L. M., \& Hellekjær, G. O. (2018). Outliers: Upper secondary school students who read better in the L2 than in L1. International Journal of Educational Research, 89(November 2017), 80-91. https://doi.org/10.1016/j.ijer.2017.10.001

Calderón, A., Arias-Estero, J.L., Meroño, L., \& Méndez-Giménez, A. (2018). Diseño y Validación del Cuestionario de Percepción del Profesorado de Educación Primaria sobre la Inclusión de las Competencias Básicas. Estudios sobre Educación, 34, 67-97. https://doi.org/10.15581/004.34.67-97

Colás, M. P., Buendía, L., \& Hernández, F. (2009). Competencias científicas para la realización de una tesis doctoral. Barcelona: Davinci.

Comi, S. L., Argentin, G., Gui, M., Origo, F., \& Pagani, L. (2017). Is it the way they use it? Teachers, ICT and student achievement. Economics of Education Review, 56, 24-39. https://doi.org/10.1016/j.econedurev.2016.11.007

Dong, X., \& Hu, J. (2019). An Exploration of Impact Factors Influencing Students' Reading Literacy in Singapore with Machine Learning Approaches. International Journal of English Linguistics, 9(5), 52. https://doi.org/10.5539/ijel.v9n5p52

Fernández-Gutiérrez, M., Gimenez, G., \& Calero, J. (2020). Is the use of ICT in education leading to higher student outcomes? Analysis from the Spanish Autonomous

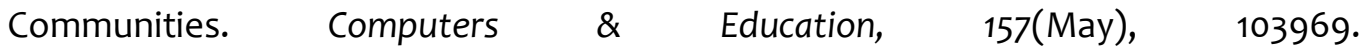
https://doi.org/10.1016/j.compedu.2020.103969

Fernández-Martín, E. (2020). Análisis de estrategias metodológicas docentes apoyadas en el uso de las TIC para fomentar el aprendizaje cooperativo del alumnado universitario en el Grado de Pedagogía. Revista Interuniversitaria de Formación del Profesorado, 95(34.2), 79-100. https://doi.org/10.47553/rifop.v34i2.77628

Gaitán, J. A., \& Piñuel, J. L. (1998). Técnicas de investigación en comunicación social. Elaboración y registro de datos. Madrid: Síntesis.

Gamazo, A., Martínez-Abad, F., Olmos-Miguelañez S., \& Rodríguez-Conde M. J. (2018). Evaluación de los factores relacionados con la eficacia escolar en PISA. Un análisis multinivel. Revista de Educación, 379, 56-84. https://doi.org/10.4438/1988-592X-RE2017-379-369

Gil, M., Cordero, J. M., \& Cristóbal, V. (2018). Las estrategias docentes y los resultados en PISA 2015. Revista de Educación, 379, 32-55. https://doi.org/10.4438/1988-592X-RE2017-379-368

Gil-Flores, J., \& García-Gómez, S. (2017). Importancia de la actuación docente frente a la política educativa regional en la explicación del rendimiento en PISA. Revista de Educación, 378, 52-77. https://doi.org/10.4438/1988-592X-RE-2017-378-361 
Giménez, G., Barrado, B., \& Arias, R. (2019). El papel del profesorado y el entorno de aprendizaje en el rendimiento de los estudiantes costarricenses: Un análisis a partir de PISA. Revista Complutense de Educacion, 30(4), 1127-1145. https://doi.org/10.5209/rced.60189

Gubbels, J., Swart, N. M., \& Groen, M. A. (2020). Everything in moderation: ICT and reading performance of Dutch 15-year-olds. Large-Scale Assessments in Education, 8(1), 1-17. https://doi.org/10.1186/s40536-020-0079-0

Harks, B., Rakoczy, K., Hattie, J., Besser, M., \& Klieme, E. (2014). The effects of feedback on achievement, interest and self-evaluation: the role of feedback's perceived usefulness. Educational 269-290. https://doi.org/10.1080/01443410.2013.785384

Huang, J., Tang, Y., He, W., \& Li, Q. (2019). Singapore's School Excellence Model and student learning: evidence from PISA 2012 and TALIS 2013. Asia Pacific Journal of Education, 39(1), 96-112. https://doi.org/10.1080/02188791.2019.1575185

Jiménez-Hernández, D., González, J. J., \& Tornel, M. (2020). Metodologías activas en la universidad y su relación con los enfoques de enseñanza. Profesorado, Revista de Currículum y Formación Del Profesorado, 24(1), 76-94. https://doi.org/10.30827/profesorado.v24i1.8173

Lau, K., \& Ho, E.S (2016). Reading Performance and Self-regulated Learning of Hong Kong Students: What We Learnt from PISA 2009. The Asia-Pacific Educational Researcher, 25, 159-171. https://doi.org/10.1007/s40299-015-0246-1

Lorenzo, F. (2016). Communicative language competence: strategies for reading literacy advance in PISA scores. Revista de Educacion, 374, 142-160. https://doi.org/10.4438/1988-592X-RE-2016-374-329

Martínez-Oviedo, I., Toledo-Toledo, G. \& Martínez-Mendoza, E. (2021). Desarrollo de un videojuego y su tablero de baile, para el aprendizaje de matemáticas básicas. Revista Electrónica Interuniversitaria de Formación del Profesorado, 24(1), 1-12. https://doi.org/10.6018/reifop.403451

MEFP (2020a). Educabase. Recuperado de https://bit.ly/39JlqtG

MEFP (2020b). Registro estatal de centros docentes no universitarios. Recuperado de https://bit.ly/2WMMiFW

Meroni, E. C., Vera-Toscano, E., \& Costa, P. (2015). Can low skill teachers make good students? Empirical evidence from PIAAC and PISA. Journal of Policy Modeling, 37(2), 308-323. https://doi.org/10.1016/j.jpolmod.2015.02.006

Murphy, L., Eduljee, N. B., Croteau, K., \& Parkman, S. (2020). Relationship between personality type and preferred teaching methods for undergraduate college students. International Journal of Research in Education and Science (IJRES), 6(1), 100-109. https://doi.org/10.46328/ijres.v6i1.690

O'Connell, M. (2019). Is the impact of SES on educational performance overestimated? Evidence from the PISA survey. Intelligence, 75, 41-47. https://doi.org/10.1016/j.intell.2019.04.005.

OCDE (2019a). PISA 2018. Programa para la evaluación internacional de los estudiantes. Informe Español. OCDE Publishing. https://bit.ly/2HPpgtE 
OCDE (2019b). PISA 2018. Technical report. OCDE Publishing. https://bit.ly/2HPszws

OCDE (2020). PISA 2018. Resultados de lectura en España. OCDE Publishing. https://bit.ly/2BJ3P9P

Perera, L. D. H., \& Asadullah, M. N. (2019). Mind the gap: What explains Malaysia's underperformance in Pisa? International Journal of Educational Development, 65(November 2017), 254-263. https://doi.org/10.1016/j.ijedudev.2018.08.010

Pérez-Pueyo, A., \& Hortigüela, D. (2020). ¿Y si toda la innovación educativa en Educación Física no es positiva? Reflexiones y consideraciones prácticas. Retos, 37, 579-587. https://bit.ly/2SpaE6u

Petko, D., Cantieni, A., \& Prasse, D. (2017). Perceived quality of educational technology matters a secondary analysis of students' ICT use, ICT-related attitudes, and PISA 2012 test scores. Journal of Educational Computing Research, 54, 1070-1091. https ://doi.org/10.1177/0735633116649373.

Rodríguez-García, A., \& Arias-Gago, A.R. (2019). Uso de metodologías activas. Un estudio comparativo entre profesores y maestros. Brazilian Journal of Development, 5(6), 5098-5111. https://bit.ly/2Zkuykk

Rodríguez-García, A., \& Arias-Gago, A.R. (2020). Revisión de propuestas metodológicas: Una taxonomía de agrupación categórica. Alteridad, 15(2), 146-160. https://doi.org/10.17163/alt.v15n2.2020.01

Rogiers, A., Van Keer, H., \& Merchie, E. (2020). The profile of the skilled reader: An investigation into the role of reading enjoyment and student characteristics. International Journal of Educational Research, 99(November 2019). https://doi.org/10.1016/j.ijer.2019.101512

Ruiz-Marín, H. (2020). ¿Cómo aprendemos? Una aproximación científica al aprendizaje y a la enseñanza. Barcelona: Graó

Sáiz-Manzanares, M.C. \& Valdivieso-León, L. (2020). Relación entre rendimiento académico y desarrollo de Estrategias de autorregulación en estudiantes universitarios. Revista Electrónica Interuniversitaria de Formación del Profesorado, 23(3), 49-65. https://doi.org/10.6018/reifop.385491

Sälzer, C., \& Roczen, N. (2018). International Journal of Development Education and Global Learning Assessing global competence in PISA 2018: Challenges and approaches to capturing a complex construct. International Journal of Development Education and Global Learning, 10(1), 6-20. https://doi.org/10.18546/IJDEGL.10.1.02

Sánchez-Fuentes, S., Martín-Almaraz, R.A., Moreno-Medina, I. \& Espada-Chavarría, R. (2018). Revisión sobre la intervención precoz en dificultades de aprendizaje relacionadas con la lectura. Revista Electrónica Interuniversitaria de Formación del Profesorado, 21(3), 35-45. http://dx.doi.org/10.6018/reifop.21.3.335171

Schaffner, E., Philipp, M., \& Schiefele, U. (2016). Reciprocal effects between intrinsic reading motivation and reading competence? A cross-lagged panel model for academic track and nonacademic track students. Journal of Research in Reading, 39(1), 19-36. https://doi.org/10.1111/1467-9817.12027

Tavsancil, E., Yildirim, O., \& Bilican, S. (2019). Direct and indirect effects of learning strategies and reading enjoyment on pisa 2009 reading performance. Eurasian Journal of Educational Research, 2019(82), 169-190. https://doi.org/10.14689/ejer.2019.82.9 
Torrpa, M., Eklund, K., Sulkunen, S., Niemi, P., \& Ahonen, T. (2017). Why do boys and girls perform differently on PISA reading in Finland? The effects of reading fluency, achievement behaviour, leisure reading and homework activity. Journal of research in reading, 41(1), 122-139. https://doi.org/10.1111/1467-9817.12103

Trigwell, K., \& Prosser, M. (2004). Development and Use of the Approaches to Teaching Inventory. Educational Psychology Review, 16(4), 409-424. https://doi.org/10.1007/s10648-004-0007-9

Van Hek, M., Kraaykamp, G., \& Pelzer, B. (2018). Do schools affect girls' and boys' reading performance differently? A multilevel study on the gendered effects of school resources and school practices. School Effectiveness and School Improvement, 29(1), 1-21. https://doi.org/10.1080/09243453.2017.1382540

Vázquez-Cano, E., De-la-Calle-Cabrera, A. M., Hervás-Gómez, C., \& López-Meneses, E. (2020). El contexto sociofamiliar y su incidencia en el rendimiento lector del estudiante en PISA. Ocnos, 19(1), 43-54. https://doi.org/10.18239/ocnos_2020.19.1.2122

Vázquez-Cano, E., Gómez-Galán, J., Infante-Moro, A., \& López-Meneses, E. (2020). Incidence of a non-sustainability use of technology on students' reading performance in Pisa. Sustainability (Switzerland), 12(2). https://doi.org/10.3390/su12020749

Wigfield, A., Gladstone, J. R., \& Turci, L. (2016). Beyond cognition: Reading motivation and reading comprehension. Child Development Perspectives, 10, 1-6. https://doi.org/10.1111/cdep.12184

Zasacka, Z., \& Bulkowski, K. (2017). Reading engagement and school achievement of lower secondary school students. Edukacja, 141(2), 78-99. https://doi.org/10.24131/3724.170205 